\title{
Determine Power Transfer Limits of An SMIB System through Linear System Analysis with Nonlinear Simulation Validation
}

\author{
Yin Li, Student Member, IEEE, Lingling Fan, Senior Member, IEEE
}

\begin{abstract}
This paper extends the materials presented in Chapter 7 of a classic textbook "Power System Analysis" [1] used in EEL 6936 Power Systems II at University of South Florida. This research examines the effect of automatic voltage regulator (AVR) and the power system stabilizer (PSS) on the power transfer level. The system analyzed in this paper includes a synchronous generator connected to an infinite bus. Their effect on stability is investigated through plotting eigenvalues of closed-loop transfer functions along with Routh-Hurwitz stability criterion examination. Three different models are considered. The basic model includes a swing equation with one order rotor flux dynamics. This model is classified as an electromagnetic model (EMT). Then, the voltage control part is added (EMT+AVR). Finally, the power system stabilizer is added to the system (EMT+AVR+PSS). These models are linearized for analysis. Nonlinear simulation results based on the nonlinear models built in Matlab/Simulink validate the analysis results.
\end{abstract}

Index Terms-Infinite bus, linear analysis, non-linear simulation, power level, AVR, PSS.

\section{INTRODUCTION}

This paper extends the materials presented in Chapter 7 of a classic textbook "Power System Analysis" [1] used in EEL 6936 Power Systems II at University of South Florida. This research examines the effect of automatic voltage regulator (AVR) and the power system stabilizer (PSS) on the power transfer level. The system analyzed in this paper includes a synchronous generator connected to an infinite bus. Their effect on stability is investigated through plotting eigenvalues of closed-loop transfer functions along with Routh-Hurwitz stability criterion examination. Three different models are considered. The basic model includes a swing equation with one order rotor flux dynamics. This model is classified as an electromagnetic model (EMT). Then, the voltage control part is added (EMT+AVR). Finally, the power system stabilizer is added to the system (EMT+AVR+PSS). These models are linearized for analysis. Nonlinear simulation results based on the nonlinear models built in Matlab/Simulink validate the analysis results.

Steady state analysis normally uses linear models [2]. To find the power transfer level limit of each model in the steady state, there are three linear models analyzed, EMT, EMT+AVR, EMT+AVR+PSS. Different initial rotor angles represent different power levels. When the required power is larger than the power transfer level, the system will be

Y. Li and L. Fan are with Dept. of Electrical Engineering, University of South Florida, Tampa FL 33620. Email: linglingfan@usf.edu. unstable. Although the analysis uses linear models, the initial value or operating point calculation requires the non-linear algebraic equations. Hence, the non-linear algebraic equations are derived first using the phasor diagram. The dynamic models are linearized to build the linear models. The stability of linear models is determined by two ways, closed-loop transfer function pole location map and Routh Hurwitz. Furthermore, the simulation section shows the dynamic responses of the corresponding non-linear models. By comparing the linear and non-linear results, the power level can be determined and the effects of AVR and PSS can be verified.

\section{LINEAR ANALYSIS}

The system considered in this paper is a generator connected to an infinite bus through a transmission line shown in Fig. 1. The synchronous generator in the system has a salient-pole rotor and its damper is negligible $\left(i_{D}=i_{Q}=0\right)$. It includes rotor resistance and inductance, $r_{f}$ and $L_{f}$. The stator impedance is represented by the $d q$ components, $L_{d}, L_{q}$, while the stator resistance is zero. The transmission line is purely inductive, $L_{L} . X$ is the impedance of the corresponding inductance.

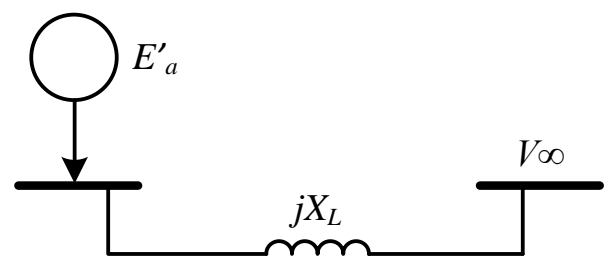

Fig. 1. A generator connected to an infinite bus.

\section{A. EMT model}

For EMT model, there are three dynamic variables, transient stator voltage, $\left|E_{a}^{\prime}\right|$, stator frequency, $\omega$, and the phase angle of the stator voltage, $\delta$. The set of differential equations of $\omega$ and $\delta$ is named swing equation. Including the differential equation of $\left|E_{a}^{\prime}\right|$, the dynamic equations can be expressed by (1).

$$
\left\{\begin{array}{l}
\frac{d\left|E_{a}^{\prime}\right|}{d t}=\left(E_{f d}-\left|E_{a}\right|\right) / T_{d o}^{\prime} \\
\frac{d \omega}{d t}=\left(P_{m}-P_{e}-D \omega_{0}(\omega-1)\right) /(2 H) \\
\frac{d \delta}{d t}=\omega_{0}(\omega-1)
\end{array}\right.
$$

where $T_{d o}^{\prime}$ is the transient time constant, $\frac{L_{f}}{r_{f}}, D$ is the damper coefficient and $H$ is proportional to the inertial of the 
generator. $E_{f d}$, is proportional to the field voltage, $v_{f}$, and is a constant input in EMT model. $P_{m}$ is the mechanical power or demanded power which is another input of the system with a small step-change. $\left|E_{a}\right|$ is the stator voltage and $P_{e}$ is the electrical power. Both of them can be represented by $\left|E_{a}^{\prime}\right|$ using phase diagram shown in Fig. 2. Thus, EMT model is considered as a third-order system.

$$
\begin{gathered}
\left|E_{a}\right|=\frac{\widetilde{X_{d}}}{\widetilde{X_{d}^{\prime}}}\left|E_{a}^{\prime}\right|+\left|V_{\infty}\right| \frac{\widetilde{X_{d}^{\prime}}-\widetilde{X_{d}}}{\widetilde{X_{d}^{\prime}}} \cos (\delta) \\
P_{e}=\frac{\left|E_{a}^{\prime}\right|\left|V_{\infty}\right|}{\widetilde{X_{d}^{\prime}}} \sin (\delta)+\frac{\left|V_{\infty}\right|^{2}}{2}\left(\frac{1}{\widetilde{X_{q}}}-\frac{1}{\widetilde{X_{d}^{\prime}}}\right) \sin (2 \delta)
\end{gathered}
$$

where $X_{d}^{\prime}$ is the transient stator impedance in $d$-axis. $\widetilde{X}$ means this impedance including $X_{L}$.

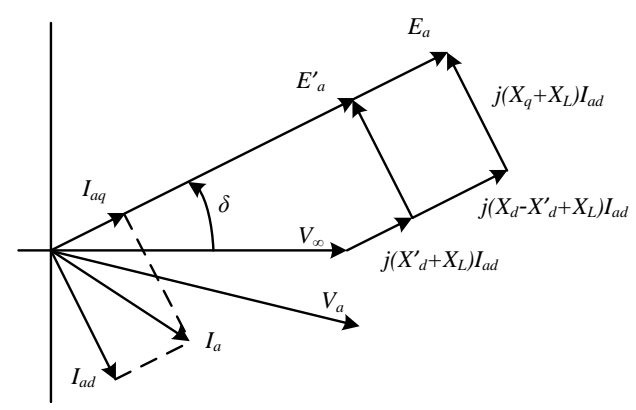

Fig. 2. Phasor diagram.

In the steady state, $\left|E_{a}^{\prime}\right|$ is only related to $E_{f d}$.

$$
\left|E_{a}^{\prime}\right|=\left(E_{f d}-V_{\infty} \cos (\delta)\right) \frac{\widetilde{X_{d}^{\prime}}}{\widetilde{X_{d}}}+V_{\infty} \cos (\delta)
$$

Based on the steady-state equations, all of initial values of the system can be calculated including $\left|E_{a}^{\prime}\right|$ and $P_{e}$, if the initial value of the phase angle of the stator voltage, $\delta^{0}$, is provided. A specific $\delta^{0}$ is corresponding to a specific $P_{e}$, so $\delta^{0}$ can be considered as the power level of the system.

Dynamic equations and steady-state equations are employed to build the non-linear model because they are non-linear. For the linear analysis of EMT model, all of them have to be linearized using linear differential equations of variables.

To linearize the dynamic equations, add a small perturbations of $\Delta \delta$ to $\delta^{0}$ to write the linear differential equation. Linearizing (2):

$$
\begin{aligned}
& \Delta\left|E_{a}\right|=\frac{\partial\left|E_{a}\right|}{\partial\left|E_{a}^{\prime}\right|} \Delta\left|E_{a}^{\prime}\right|+\frac{\partial\left|E_{a}\right|}{\partial \delta} \Delta \delta \\
& \Delta\left|E_{a}\right|=\frac{\widetilde{X_{d}}}{\widetilde{X_{d}^{\prime}}} \Delta\left|E_{a}^{\prime}\right|+\left(\frac{\widetilde{X_{d}}}{\widetilde{X_{d}^{\prime}}-1}\right) V_{\infty} \sin (\delta) \Delta \delta \\
& \Delta\left|E_{a}\right|=\frac{1}{k_{3}} \Delta\left|E_{a}^{\prime}\right|+k_{4} \Delta \delta
\end{aligned}
$$

Substituting (5) into $\left|E_{a}^{\prime}\right|$ differential equation of (1) to find the linear relationship between $\Delta\left|E_{a}^{\prime}\right|$ and $\Delta \delta$,

$$
\begin{aligned}
T_{d o}^{\prime} \frac{d \Delta\left|E_{a}^{\prime}\right|}{d t} & =\Delta E_{f d}-\frac{1}{k_{3}} \Delta\left|E_{a}^{\prime}\right|-k_{4} \Delta \delta \\
\Delta\left|E_{a}^{\prime}\right| & =\frac{k_{3}}{\left(T_{d o}^{\prime} k_{3}\right) s+1}\left(\Delta E_{f d}-k_{4} \Delta \delta\right)
\end{aligned}
$$

Using the same way to linearize (3),

$$
\begin{aligned}
& \Delta P_{e}=\frac{\partial P_{e}}{\partial\left|E_{a}^{\prime}\right|} \Delta\left|E_{a}^{\prime}\right|+\frac{\partial P_{e}}{\partial \delta} \Delta \delta \\
& \Delta P_{e}=\frac{V_{\infty}}{\widetilde{X_{d}^{\prime}}} \sin (\delta) \Delta\left|E_{a}^{\prime}\right|+ \\
& \left.\qquad \frac{\left|E_{a}^{\prime}\right|\left|V_{\infty}\right|}{\widetilde{X_{d}^{\prime}}} \cos (\delta)+\left|V_{\infty}\right|^{2}\left(\frac{1}{\widetilde{X_{q}}}-\frac{1}{\widetilde{X_{d}^{\prime}}}\right) \cos (2 \delta)\right] \Delta \delta \\
& \Delta P_{e}=k_{2} \Delta\left|E_{a}^{\prime}\right|+T \Delta \delta
\end{aligned}
$$

Linearizing the swing equations by substituting (7) into them,

$$
\left\{\begin{aligned}
\Delta \dot{\omega} & =\left(\Delta P_{m}-\left(k_{2} \Delta\left|E_{a}^{\prime}\right|+T \Delta \delta\right)-D \omega_{0} \Delta \omega\right) /(2 H) \\
\Delta \dot{\delta} & =\omega_{0} \Delta \omega
\end{aligned}\right.
$$

Combining them to obtain the second-order transfer function,

$$
\begin{aligned}
\frac{2 H}{\omega_{0}} \ddot{\Delta \delta} & =\Delta P_{m}-k_{2} \Delta\left|E_{a}^{\prime}\right|-T \Delta \delta-D \dot{\Delta \delta} \\
\left(M s^{2}+D s+T\right) \Delta \delta & =\Delta P_{m}-k_{2} \Delta\left|E_{a}^{\prime}\right| \\
\frac{\Delta \delta}{\Delta P_{m}-k_{2} \Delta\left|E_{a}^{\prime}\right|} & =\frac{1}{M s^{2}+D s+T}
\end{aligned}
$$

After linearizing the dynamic equations of EMT model, its linearized model is designed using block diagram shown in Fig. 3. $\Delta P_{m}$ is considered as the input and $\Delta \delta$ is the output. Because of the constant $E_{f d}$ in this system, $\Delta E_{f d}$ is zero. Based on Fig. 3, the closed-loop transfer function of this

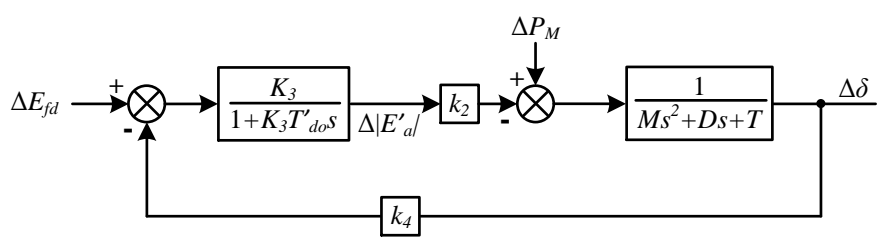

Fig. 3. Block diagram of linearized EMT system.

system can be derived to find the pole locations.

$$
\begin{aligned}
T_{E M}(s) & =\frac{1}{M s^{2}+D s+T} \\
T_{E M T}(s) & =\frac{T_{E M}(s)}{1-T_{E M}(s) * k_{4} * \frac{k 3}{\left(T_{d o}^{\prime} k_{3}\right) s+1} k_{2}} \\
& =\frac{\left(T_{d o}^{\prime} k_{3}\right) s+1}{\left.\left(T_{d o}^{\prime} k_{3} s+1\right)\left(M s^{2}+D s+T\right)-k_{2} k_{3} k_{4}\right)}
\end{aligned}
$$

When $\delta^{0}$ is given, the gains and coefficients of $T_{E M T}(s)$ can be calculated; then, MATLAB code $\operatorname{pzplot}\left(T_{E M T}(s)\right)$ is used to plot the pole-zero locations. If there is any pole located in RHP, the system will be unstable. Therefore, the largest $\delta^{0}$ which makes the system stable can be considered as the power 
level of this system. To find the power level, $\delta^{0}$ is increased from 0 to $90^{\circ}$. The increment is $1^{\circ}$. Based on the closed-loop transfer function, one value of $\delta^{0}$ will generate three poles. Fig. 4 plots all of pole locations for the whole range of $\delta^{0}$. It is observed that when $\delta^{0}$ is over $81^{\circ}$, the pole aligned on the real axis is located on RHP, so $81^{\circ}$ can be considered as the power level of EMT model.

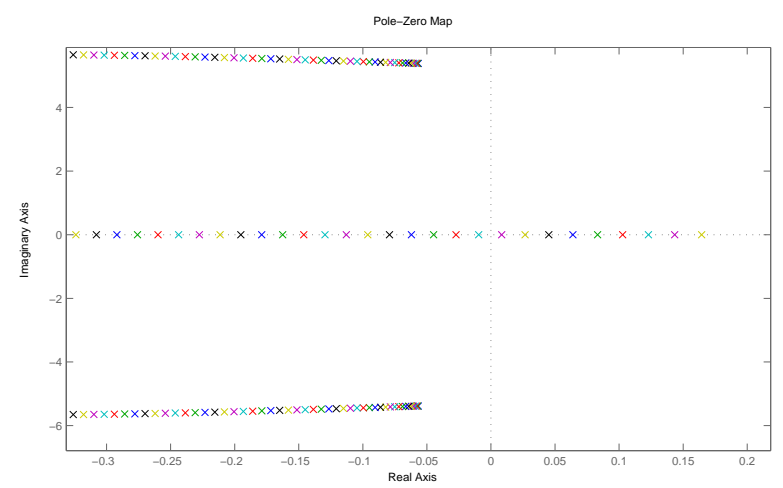

Fig. 4. Pole-zero locations of EMT model. From $0^{\circ}$ to $90^{\circ}$

The power level, $81^{\circ}$, also can be verified by the Routh Hurwitz.

$$
\begin{array}{c|ccc}
s^{3} & T_{d o}^{\prime} k^{3} M & T_{d o}^{\prime} k_{3} T+D & 0 \\
s^{2} & T_{d o}^{\prime} k_{3} D+M & T-k_{2} k_{3} k_{4} & 0 \\
s^{1} & K_{1} & 0 \\
s^{0} & T-k_{2} k_{3} k_{4} & &
\end{array}
$$

where $K_{1}=T_{d o}^{\prime} k_{3} T+D-\frac{\left(T_{d o}^{\prime} k^{3} M\right)\left(T-k_{2} k_{3} k_{4}\right)}{\left(T_{d o}^{\prime} k_{3} D+M\right)}$. Based on the initial value calculation, $K_{1}$ is always larger than 0 . Therefore, the sign of $T-k_{2} k_{3} k_{4}$ determines if the system stable or not. It is 0.0067 at $\delta^{0}=81^{\circ}$ while it is -0.0058 at $\delta^{0}=82^{\circ}$.

\section{B. EMT+AVR model}

When AVR is added to EMT model, $E_{f d}$ will not be a constant input, but a variable which is controlled by the terminal voltage. $E_{f d}$ is generated by an exciter, so the input of the exciter is the error between the terminal voltage, $V_{a}$, and its reference, $V_{\text {ref }}$. Certainly, $V_{a}$ is dependent on the demanded power, $P_{m}$ or $\delta^{0}$. Before linearizing the system, the equations for calculating the initial values of EMT+AVR model should be derived.

If the power factor and $\delta^{0}$ are provided, the stator current, $I_{a}$, can be calculated because $V_{\infty}$ is constant. In this paper, reactive power, $Q$, is zero, so power factor is 1 and $I_{a}$ is aligned on $V_{\infty}$.

$$
\begin{aligned}
a^{\prime} & =V_{\infty}+j \widetilde{X_{q}} I_{a} \\
I_{a} & =\frac{V_{\infty} * \tan (\delta)}{\widetilde{X}_{q}}
\end{aligned}
$$

Based on Fig. 2, $E_{a}$ can be expressed by $I_{a}, V_{\infty}$, and $\delta^{0}$.
In addition, $E_{f d}$ is equal to $E_{a}$ in steady state (1).

$$
\begin{aligned}
E_{a} & =V_{q}-\widetilde{X_{d}} I_{a d} \\
E_{a} & =V_{\infty} \cos \left(\delta^{0}\right)+\widetilde{X_{d}} I_{a} \sin (\delta) \\
E_{f d} & =\left|E_{a}\right|
\end{aligned}
$$

If $E_{f d}$ is calculated, $\left|E_{a}^{\prime}\right|$ is obtained (4); then, the terminal voltage is calculated by $\left|E_{a}^{\prime}\right|$ [1].

$$
\begin{aligned}
V_{a q} & =\frac{X_{L}}{\widetilde{X}_{d}^{\prime}}\left|E_{a}^{\prime}\right|+\frac{X_{d}^{\prime}}{\widetilde{X}_{d}^{\prime}} V_{\infty} \cos (\delta) \\
V_{a d} & =-\frac{X_{q}}{\widetilde{X}_{q}} V_{\infty} \sin (\delta) \\
\left|V_{a}\right| & =\sqrt{\left(V_{a d}\right)^{2}+\left(V_{a q}\right)^{2}} \\
V_{\text {ref }} & =\frac{E_{f d}}{k_{A}}+\left|V_{a}\right|
\end{aligned}
$$

where $k_{A}$ is the amplify gain. In this paper, its value is 25 .

Before linearizing the EMT+AVR model, there are two assumptions which are made to simplify the model. It is assumed that the voltage control system is very fast, so the time constant of the control loop is zero; the exciter is assumed as a pure gain, $G_{e}(s)=k_{A}$.

Because $E_{f d}$ is not a constant in EMT+AVR model and it is controlled by $\left|V_{a}\right|, V_{a}$ has to be linearized by two variables, $\left|E_{a}^{\prime}\right|$ and $\delta$.

$$
\begin{aligned}
\Delta\left|V_{a}\right| & =\frac{\partial\left|V_{a}\right|}{\partial \delta} \Delta \delta+\frac{\partial\left|V_{a}\right|}{\partial \delta} \Delta\left|E_{a}^{\prime}\right| \\
\Delta\left|V_{a}\right| & =-\left|V_{\infty}\right|\left(\frac{X_{d}^{\prime} V_{a q}}{\widetilde{X_{d}^{\prime}}\left|V_{a}\right|} \sin (\delta)+\frac{X_{q} V_{a d}}{\widetilde{X_{q}}\left|V_{a}\right|} \cos (\delta)\right) \Delta \delta \\
& +\frac{X_{L} V_{a q}^{0}}{\widetilde{X_{d}^{\prime}}\left|V_{a}\right|^{0}} \Delta\left|E_{a}^{\prime}\right| \\
\Delta\left|V_{a}\right| & =k_{5} \Delta \delta+k_{6} \Delta\left|E_{a}^{\prime}\right|
\end{aligned}
$$

After adding the linearized AVR part to EMT model, Fig. 5 shows the block diagram of EMT+AVR model. $\Delta P_{m}$ is the input, $\Delta \delta$ is the output, and $\Delta V_{r e f}$ is normally zero.

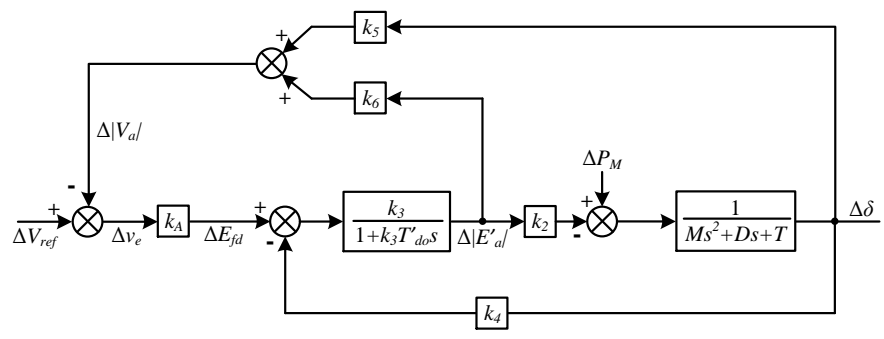

Fig. 5. Block diagram of linearized EMT+AVR system.

To reduce Fig. 5 to a single loop, the feedback transfer function is derived first.

$$
\begin{aligned}
\Delta\left|E_{a}^{\prime}\right| & =\frac{k_{3}}{T_{d o}^{\prime} k_{3} s+1}\left[-k_{4} \Delta \delta-k_{A}\left(k_{5} \Delta \delta+k_{6} \Delta\left|E_{a}^{\prime}\right|\right)\right] \\
\frac{\Delta\left|E_{a}^{\prime}\right|}{\Delta \delta} & =\frac{-k_{3}\left(k_{4}+k_{A} k_{5}\right)}{T_{d o}^{\prime} k_{3} s+k_{3} k_{A} k_{6}+1} \\
& =-\frac{1}{k_{2}} \frac{a}{b s+c}
\end{aligned}
$$


where $a=k_{2} k_{3}\left(k_{4}+k_{e} k_{5}\right), b=k_{3} T_{d o}, c=k_{3} k_{e} k_{6}+1$. Using (15) to derive the closed-loop transfer function:

$$
\begin{aligned}
T_{A V R}(s) & =\frac{T_{E M}(s)}{1-T_{E M}(s) * \frac{a}{b s+c}} \\
& =\frac{b s+c}{\left(M s^{2}++D s+T\right)(b s+c)-a} \\
& =\frac{b s+c}{M b s^{3}+(D b+M c) s^{2}+(T b+D c) s+T c-a}
\end{aligned}
$$

Theoretically, EMT+AVR system will be unstable if supplying a high active power. It is determined by the same way, Pole-zero location. Based on $T_{A V R}(s)$, there are three poles and one zero with a specific $\delta_{0}$. For observing and analyzing easily, Fig. 6 only shows the poles crossing the $j \omega$ axis and the range of $\delta^{0}$ is from $40^{\circ}$ to $89^{\circ}$. It's observed that the thirteenth pole is on RHP, so the power level of EMT+AVR model is $51^{\circ}$. This also can be proved by Routh Hurwitz.

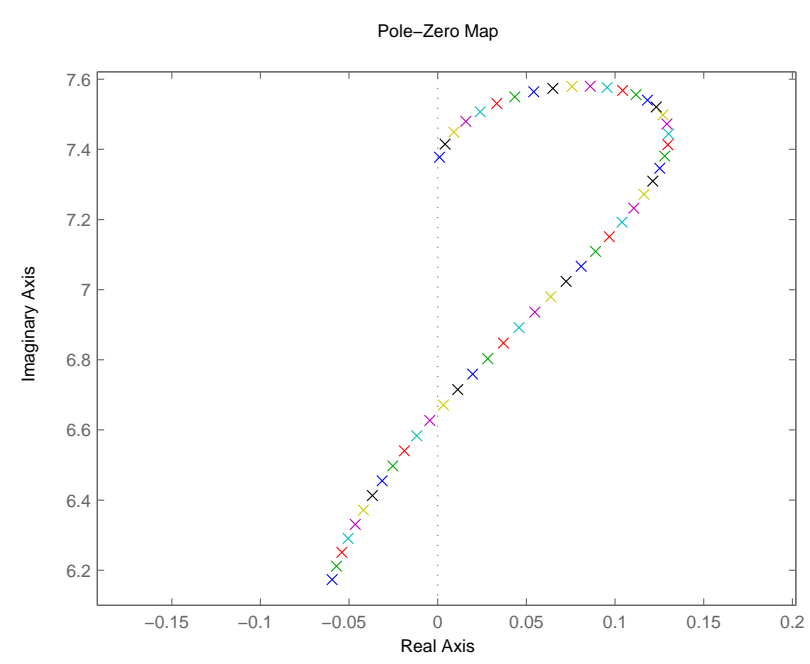

Fig. 6. Pole-zero locations of EMT+AVR model. From $40^{\circ}$ to $89^{\circ}$

$$
\begin{array}{c|ccc}
s^{3} & M b & T b+D c & 0 \\
s^{2} & D b+M c & T c-a & 0 \\
s^{1} & K_{2}=T b+D c-\frac{(M b)(T c-a)}{D b+M c} & 0 & \\
s^{0} & T c-a &
\end{array}
$$

Based on the calculation, $K_{2}$ is 0.0037 at $\delta_{0}=51^{\circ}$ while it is -0.0027 at $\delta_{0}=52^{\circ} . T c-a$ is always larger than 0 . Although AVR part can make the system supply more power, it also reduces $\delta^{0}$ of the system.

\section{III. $\mathrm{EMT}+\mathrm{AVR}+\mathrm{PSS}$}

To solve the samll $\delta^{0}$ of EMT+AVR model, PSS part is added to stabilize the system. Using linear analysis, PSS provides two zeros near the original point and one pole to change the root locus of EMT+AVR model, so the block diagram of EMT+AVR+PSS model should be like the figure in Fig. 7.

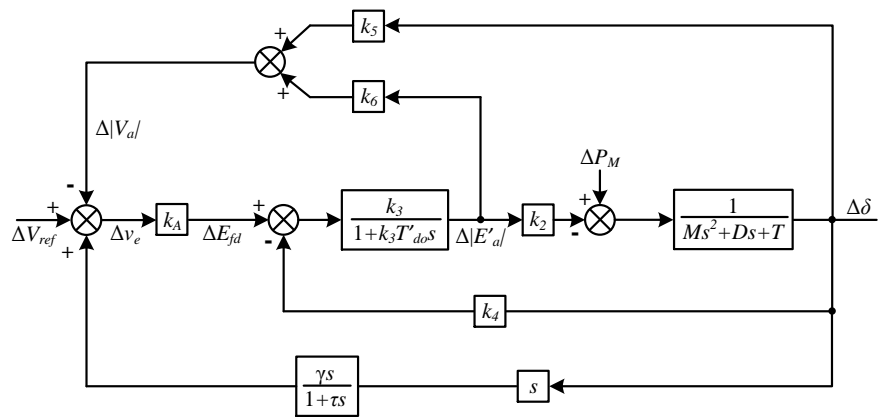

Fig. 7. Block diagram of linearized EMT+AVR+PSS system.

Using the same to derive the closed-loop transfer function of EMT+AVR+PSS model. The feedback transfer function is:

$$
\begin{aligned}
\Delta\left|E_{a}^{\prime}\right| & =\frac{k_{3}}{T_{d o}^{\prime} k_{3} s+1}\left[k_{e}\left(\frac{\gamma s^{2}}{\tau s+1} \Delta \delta-k_{5} \Delta \delta-k_{6} \Delta\left|E_{a}^{\prime}\right|\right)\right] \\
& -\frac{k_{3}}{T_{d o}^{\prime} k_{3} s+1} k_{4} \Delta \delta \\
\frac{\Delta\left|E_{a}^{\prime}\right|}{\Delta \delta} & =\frac{k_{3} k_{e} \gamma s^{2}-\tau k_{3}\left(k_{5} k_{e}+k_{4}\right) s-k_{3}\left(k_{5} k_{e}+k_{4}\right)}{\tau T_{d o}^{\prime} k_{3} s^{2}+\left(\tau k_{3} k_{6} k_{e}+\tau+T_{d o}^{\prime} k_{3}\right) s+k_{3} k_{6} k_{e}+1} \\
\frac{\Delta\left|E_{a}^{\prime}\right|}{\Delta \delta} & =\frac{1}{k_{2}} \frac{f s^{2}-\tau a s-a}{\tau b s^{2}+e s+c}
\end{aligned}
$$

where $\gamma$ and $\tau$ are the coefficients of PSS. $e=\tau k_{3} k_{6} k_{e}+\tau+$ $T_{d o}^{\prime} k_{3}$ and $f=k_{3} k_{e} \gamma k_{2}$. The closed-loop transfer function is written:

$$
\begin{aligned}
T_{P S S}(s) & =\frac{T_{E M}(s)}{1+T_{E M}(s) \frac{f s^{2}-\tau a s-a}{\tau b s^{2}+e s+c}} \\
& =\frac{\tau b s^{2}+e s+c}{\left(M s^{2}+D s+T\right)\left(\tau b s^{2}+e s+c\right)+f s^{2}-\tau a s-a}
\end{aligned}
$$

Factoring the denominator of $T_{P S S}(s): M \tau b s^{4}+(M e+$ $D \tau b) s^{3}+(M c+D e+T \tau b+f) s^{2}+(D c+T e-\tau a) s+(T c-a)$. Based on Fig. 8, PSS makes the poles near $j \omega$ axis locate on LHP when $\delta^{0}$ is increased from $1^{\circ}$ to $89^{\circ}$. Because $\delta^{0}$ cannot be $90^{\circ}$ and the increment is $1^{\circ}$, the power level can be considered to approaching $90^{\circ}$. Using Routh Hurwitz to prove it:

$$
\begin{array}{c|cccc}
s^{4} & M \tau b s & M c+D e+T \tau b+f & T c-a & 0 \\
s^{3} & M e+D \tau b & D c+T e-\tau a & 0 & 0 \\
s^{2} & K_{3} & T c-a & 0 & \\
s^{1} & K_{4} & 0 & & \\
s^{0} & T c-a & &
\end{array}
$$

where $K_{3}==M c+D e+T \tau b+f-\frac{(M \tau b)(D c+T e-\tau a)}{M e+D \tau b}, K_{4}=$ $D c+T e-\tau a-\frac{(M e+D \tau b)(T c-a)}{K_{3}}$. Based on the calculation, $K_{3}$ and $K_{4}$ are always larger than 0 .

\section{Simulation Results From Non-Linear Models}

To verify the results from the linear models, the corresponding non-linear models are designed and simulated in MATLAB/Simulink. The screen copy is shown in Fig. 9. The model building exploits the vector feature of MATLAB. For example, the output of the integrator is the vector of the three 


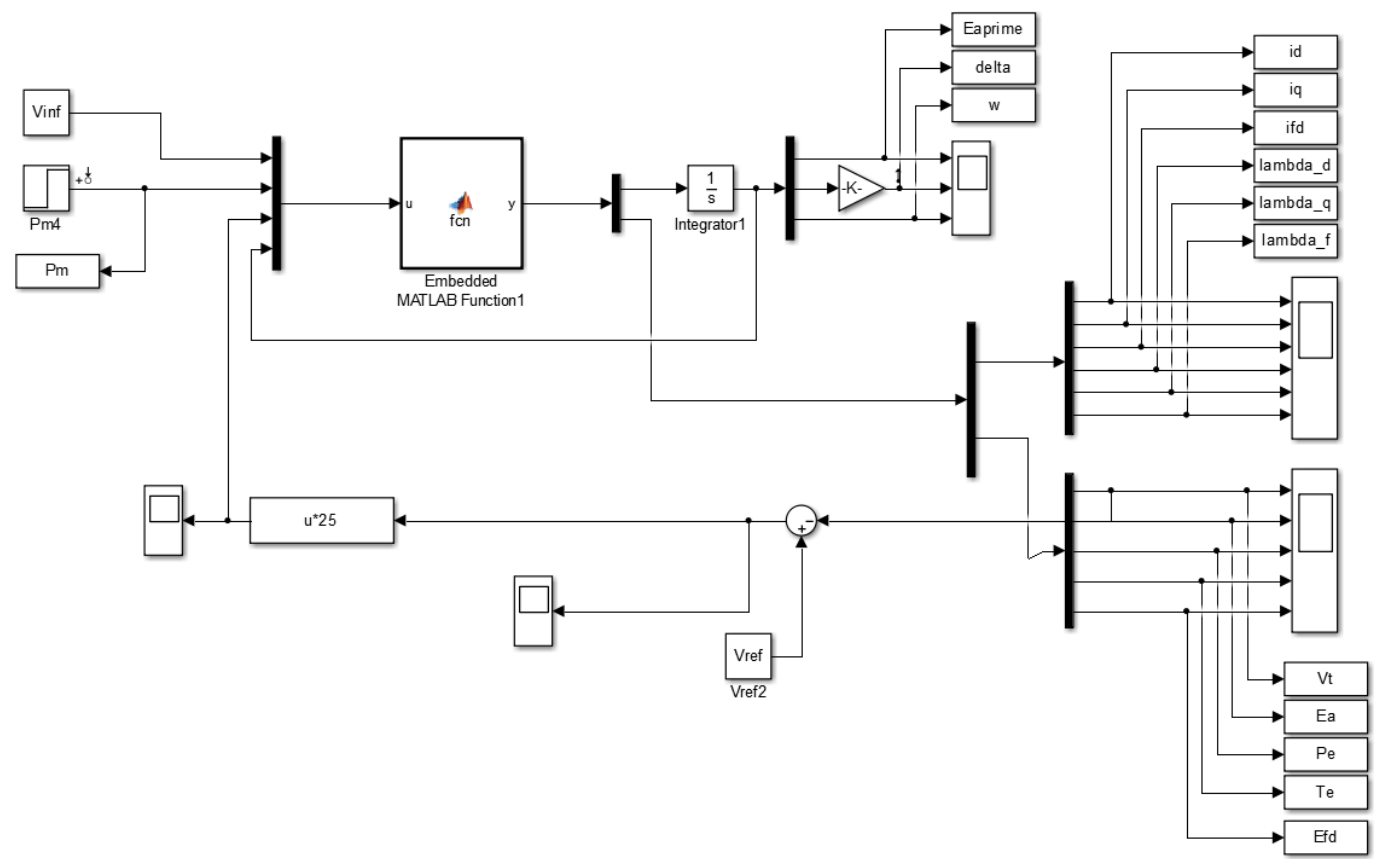

Fig. 9. Matlab/Simulink simulation blocks.

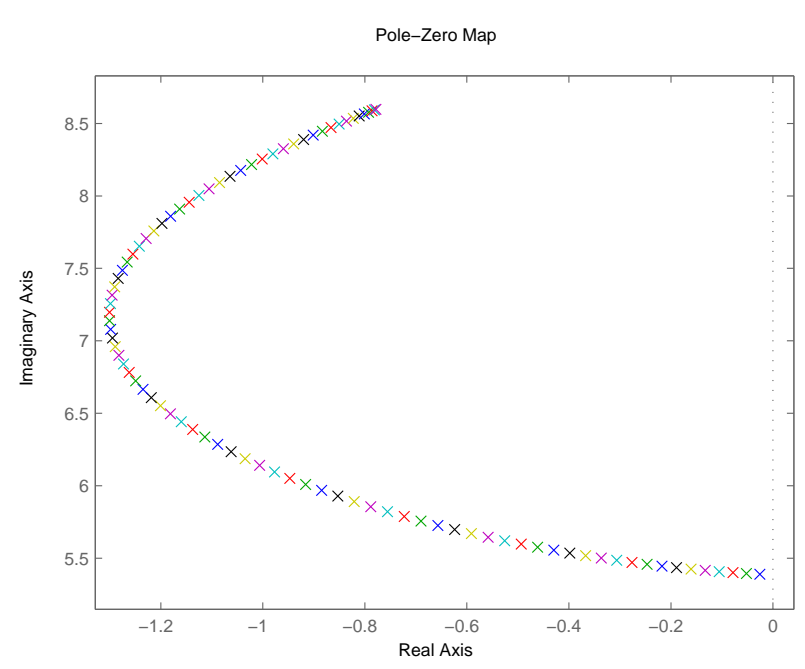

Fig. 8. Pole-zero locations of EMT+AVR+PSS model. From $1^{\circ}$ to $89^{\circ}$

state variables $E_{a}^{\prime}, \delta, \omega$. The input of the integrator comes from computation of their derivatives, which is also a vector. In addition, the embedded MATLAB function also has a vector as input and a vector as an output. Therefore, mux and demux blocks are frequently used in this model building. Such model building technique has been detailed documented in [3] to build an induction machine. The model building technique has been applied in variety of system building, e.g., a doubly fed induction generator with square wave input from rotor [4], wind farm series compensated interconnected system for subsynchronous resonance analysis [5]. This technique leads to a very concise expression.

The non-linear models are based on the differential equa-
TABLE I

PARAMETER OF THE SYSTEM.

\begin{tabular}{|l|c|c|}
\hline \multicolumn{3}{|c|}{ Table 1 } \\
\hline Parameter & Values & Value(p.u.) \\
\hline Rating Power & $835 \mathrm{MVA}$ & 1 \\
\hline Line to line Voltage & $26 \mathrm{kV}$ & 1 \\
\hline Speed & $3600 \mathrm{r} / \mathrm{min}$ & 1 \\
\hline$X_{d}, X_{q}$ & $0.8096 \Omega, 0.5667 \Omega$ & $1,0.7$ \\
\hline$r_{f}, X_{f d}$ & $0.00011 \Omega, 0.0810 \Omega$ & $0.05,0.1$ \\
\hline$X_{L}, X_{d}^{\prime}$ & $0.4048 \Omega, 0.1619 \Omega$ & $0.5,0.2$ \\
\hline$H$ & $5.6 \mathrm{~s}$ & $5.6 \mathrm{s.pu}$. \\
\hline
\end{tabular}

tions (1). Before simulating non-linear models, the initial values should be calculated; then giving a small step change in $P_{m}$ to check the system's stability.

For EMT model, the required initial values are three dynamic variables, $E_{a}^{\prime}, \delta, \omega$, one input with step change, $P_{m}$, and two constant inputs, $E_{f d}, V_{\infty}$. Because of the synchronous generator, $\omega$ should be 1p.u. at steady state. $E_{f d}$ and $V_{\infty}$ are set as 1p.u. $\delta$ should be $\delta^{0}$ to find the power level. $E_{a}^{\prime}$ and $P_{m}$ are calculated based on (12), (3). The generator's parameters are list in Table1. Although EMT+AVR model and EMT+AVR+PSS model have different block diagrams of linear models, the initial value calculations are the same. For EMT+AVR model, $\omega$ and $V_{\infty}$ are still 1p.u. but $V_{\text {ref }}$ replaces $E_{f d}$ as the constant input of the system. $E_{a}^{\prime}, P_{m}$ and $V_{r e f}$ are calculated by (3)-(4), (11)-(14).

\section{A. EMT model}

Based on the linear analysis, the power level of EMT model is $81^{\circ}$, so the initial value of $\delta^{0}$, is set as $81^{\circ}$ and one more degree than the power level, $82^{\circ}$. To avoid that a large step change will cause the system unstable, there is 0.00001 p.u. 
power added to $P_{m}$ at $2 \mathrm{~s}$. Fig. 10 shows the step response of one dynamic variable, $\delta$. Although $\delta$ responses very slowly in both of situation, it is obviously that $\delta$ is approaching a steady state at $\delta^{0}=81^{\circ}$ while it rises faster and faster at $\delta^{0}=82^{\circ}$. Hence, it is proved that the power level of EMT model is $\delta^{0}=81^{\circ}$. In addition, the pole which is very closed to $j \omega$ axis does not have the imaginary part based on Fig. 4 , so the step response of $\delta$ does not have the oscillation. Fig. 10 also proves this point.
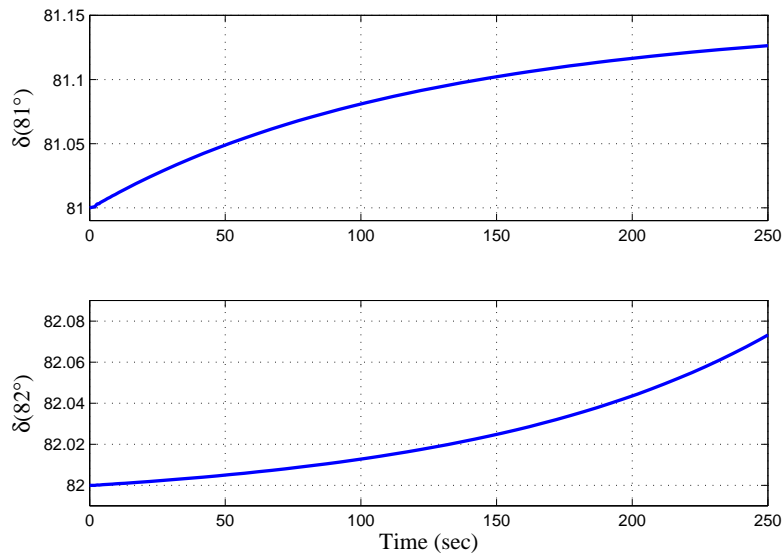

Fig. 10. The step response of $\delta$ in EMT model. Upper plot: $\delta^{0}=81^{\circ}$; lower plot: $\delta^{0}=82^{\circ}$.

\section{B. EMT+AVR model}

For the obvious difference, $\delta^{0}$ has two initial values, $51^{\circ}$ and $55^{\circ}$. The step change in $P_{m}$ of EMT+AVR model is not very sensitive, so the step change is 0.01 p.u. and happens at $2 \mathrm{~s}$. Based on Fig. 11, although the step response of $\delta$ has a large oscillation in two plots, it is observed that the oscillation is becoming smaller at $\delta^{0}=51^{\circ}$ while it is becoming larger at $\delta^{0}=55^{\circ}$. The reason causing the damping is that the pole which crosses $j \omega$ axis has an imaginary part in EMT+AVR model. Therefore, Fig. 11 does not only prove that the power level of EMT+AVR model is $51^{\circ}$, but also proves which pole causes the system unstable.
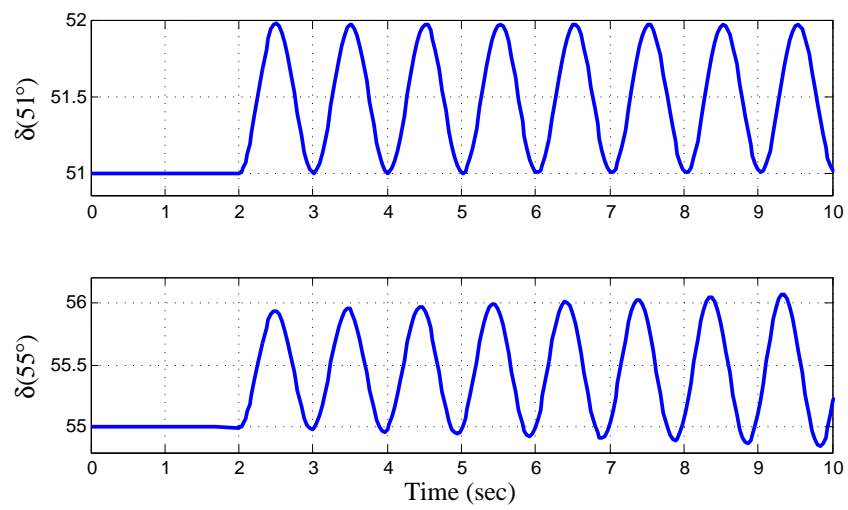

Fig. 11. The step response of $\delta$ in EMT + AVR model. Upper plot: $\delta^{0}=51^{\circ}$; lower plot: $\delta^{0}=55^{\circ}$.

\section{C. $E M T+A V R+P S S$ model}

It is already known that EMT+AVR+PSS model is always stable when $\delta^{0}$ is under $90^{\circ}$, so it is only required to check the stability of the system at $\delta^{0}=89^{\circ}$. The step change is still 0.01 p.u. at 2 s. Fig. 12 shows that the system is stable at $\delta^{0}=89^{\circ}$ and the step response is very fast. It proves that the power level of EMT+AVR+PSS model is $89^{\circ}$ and PSS provide a supplemental damping to cancel the oscillations from AVR.

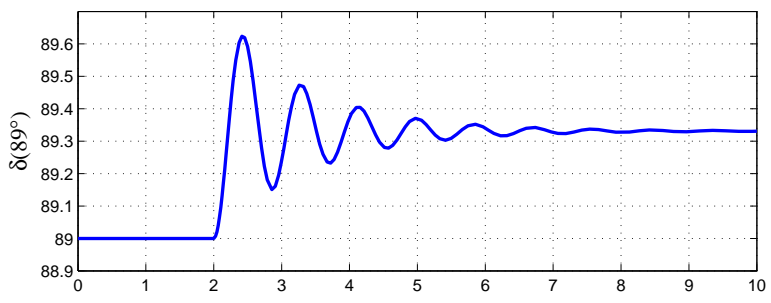

Fig. 12. The step response of $\delta$ in EMT+AVR+PSS model. $\delta^{0}=89^{\circ}$.

\section{CONCLUSION}

This paper uses the linear analysis to find the power level of the typically power system, a synchronous generator connected to an infinite bus. Moreover, it determines the power levels of this system which is added by the voltage control, AVR, and the stabilizer, PSS. The simulation results of the nonlinear models verify all of three power levels analyzed from the linear models. After the analysis and the verification, there are three points concluded. One is that the power level of this system cannot be equal or larger than $90^{\circ}$. The second is that although AVR can make the generator supply more power, the power transfer angle is its shortage. It means that if there is a large active power transfered, the system will be unstable. Last point, PSS is very useful to stabilize the system when there is higher power required to transfer. Finally, a complete power generation system should include both of AVR and PSS parts.

\section{REFERENCES}

[1] A. R. Bergen and V. Vittal, "Power systems analysis (second edition)," in Prentice Hall, 1999.

[2] P. Kundur, "Power system stability and control," in McGraw-Hill, 1994.

[3] Z. Miao and L. Fan, "The art of modeling and simulation of induction generator in wind generation applications using high-order model," Simulation Modelling Practice and Theory, vol. 16, no. 9, pp. 1239-1253, 2008.

[4] L. Fan, Z. Miao, S. Yuvarajan, and R. Kavasseri, "Hybrid modeling of dfigs for wind energy conversion systems," Simulation Modelling Practice and Theory, vol. 18, no. 7, pp. 1032-1045, 2010.

[5] L. Fan, R. Kavasseri, Z. L. Miao, and C. Zhu, "Modeling of dfig-based wind farms for ssr analysis," Power Delivery, IEEE Transactions on, vol. 25, no. 4, pp. 2073-2082, 2010. 